\title{
Probing the magnetic field structure in the filamentary cloud IC5146
}

\author{
Jia-Wei Wang ${ }^{1}$, Chakali Eswaraiah ${ }^{1,2,4}$, Shih-Ping Lai ${ }^{1}$, D. P. \\ Clemens $^{3}$, Wen-Ping Chen ${ }^{4}$, and Anil K. Pandey ${ }^{2}$ \\ ${ }^{1}$ Institute of Astronomy \& Department of Physics, National Tsing Hua University, \\ Hsinchu 30013, Taiwan \\ email: noonethere94@hotmail.com; slai@phys.nthu.edu.tw \\ ${ }^{2}$ Aryabhatta Research Institute of Observational-Sciences (ARIES), India \\ ${ }^{3}$ Institute for Astrophysical Research, Boston University, 725 Commonwealth Avenue, Boston, \\ MA 02215, USA \\ ${ }^{4}$ Institute of Astronomy, National Central University, Taiwan
}

\begin{abstract}
IC5146 is one of the filamentary clouds observed in Herschel Gould Belt Survey. Here we present our polarization observations toward IC5146 taken with AIMPOL, TRIPOL and Mimir. Our results reveal that the large scale structure of magnetic field is well perpendicular to the main filament, but is likely parallel to the sub-filaments, which are structure extended out from the main filaments. We have also conducted CO observations to reveal the gas kinematics along the filaments or magnetic field; this result suggests the gas is possibly confined by magnetic field in most of the region until reaching supercritical. Based on our results, we suggests that at least four types of filaments can be found in IC5146, behaving different physical properties and consistent with different formation mechanisms. Our conclusions reveal that filaments are a combination of a variety types of objects.
\end{abstract}

Keywords. ISM: clouds, ISM: magnetic fields, ISM: structure, ISM: evolution, polarization

\section{The Four Types of Filaments in IC5146}

We have performed optical and infrared polarization observations toward IC5146 filamentary cloud in R, i, H and $\mathrm{K}$ band (Fig. 1). The filaments in IC5146 show four different types of conditions, possibly due to the evolution with different mechanisms

(a) Type I Main-Filament: The main-filaments are perpendicular to a ordered large-scale B-field while nearby material is likely accumulated toward the main-filament along B-field, consistent with Nakamura \& Li (2008) model. In IC5146, the main-filament consists of both thermally subcritical region (eastern) and supercritical region (western). The latter is likely gravitationally fragmented along B-field, and thus appears as a bundle of filaments paralleled to each others.

(b) Type II Accretion Filament: The filaments parallel to B-field are commonly seen around a main-filament, also known as "striations". These filaments have velocity gradient along Bfield toward main-filaments. Therefore they are considered to be the accretion flows to replenish material in main-filaments (e.g. Palmeirim et al.2013).

(c) Type III Hub-Filament: Myers et al.(2009) shows that young stellar groups are often associated with the dense "hub" with radiation filaments instead of collimated orientations. In IC5146, these systems can be found in the thermally supercritical main-filament, where our ${ }^{13} \mathrm{CO}$ data shows that gas in nearby filaments is flowing toward the hubs (Fig. 2).

(d) Type IV Compressed Filament: Filaments can also be form via the compression of shock, flows or turbulence (Padaon et al.2001). In IC5146, the filaments inside the HII region is likely formed due to the compression of HII bubbles, and thus perform a shell-like morphology. 


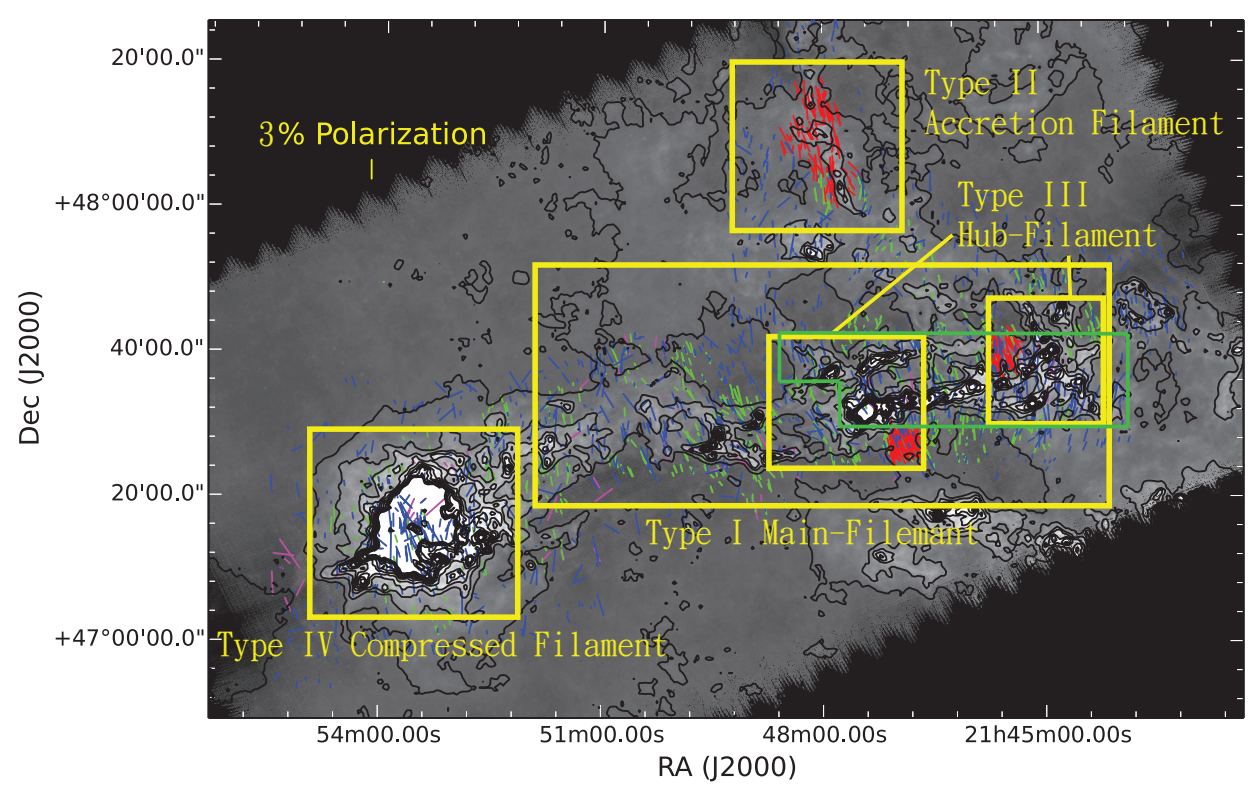

Figure 1. Polarization maps overlaid on Herschel $250 \mu \mathrm{m}$ image. The yellow boxes indicate the four types of filaments that would be discussed, and the green box label the region of Fig. 2 . The red, green, blue and magenta are the data in R, i, H and $\mathrm{K}$ band, respectively.

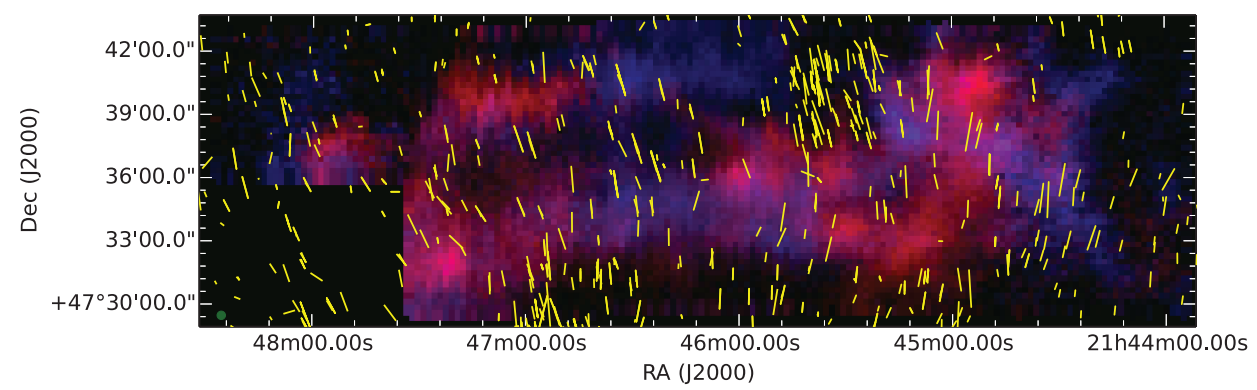

Figure 2. ${ }^{13} \mathrm{CO} \mathrm{J}=2-1$ emission toward The Western Part of Main-Filament. Redshifted ${ }^{13} \mathrm{CO}$ $\mathrm{J}=2-1$ emission integrated from $V_{L S R}=3.5 \mathrm{~km} / \mathrm{s}$ to $5 \mathrm{~km} / \mathrm{s}$ is shown as red and the blueshifted component integrated from $V_{L S R}=2 \mathrm{~km} / \mathrm{s}$ to $3.5 \mathrm{~km} / \mathrm{s}$ is shown as blue. Yellow vectors are our poalrzation data.

\section{References}

Arzoumanian, D., André, P., Didelon, P., et al.2011, A\& A, 529, L6

Myers, P. C. 2009, ApJ, 700, 1609

Nakamura, F., \& Li, Z. 2008, ApJ, 687, 354

Padoan, P., Juvela, M., Goodman, A. A., \& Nordlund, Å. 2001, ApJ, 553, 227

Palmeirim, P., André, P., Kirk, J., et al.2013, A\& A, 550, A38 